\title{
Photocatalysts for Organics Degradation
}

\author{
Barbara Bonelli ${ }^{1, *(\mathbb{C})}$, Maela Manzoli ${ }^{2}$, , Francesca S. Freyria ${ }^{1,3(\mathbb{C})}$ and Serena Esposito ${ }^{1(\mathbb{C})}$ \\ 1 Institute of Chemistry, Department of Applied Science and Technology; PoliTO BiomED Interdepartmental \\ Lab, Politecnico di Torino, 10129 Torino, Italy; francesca.freyria@polito.it (F.S.F.); \\ serena_esposito@polito.it (S.E.) \\ 2 Department of Drug Science and Technology, Università degli Studi di Torino, Via Pietro Giuria 9, \\ 10125 Torino, Italy; maela.manzoli@unito.it \\ 3 Department of Chemistry, Massachusetts Institute of Technology, Cambridge, MA 02139, USA \\ * Correspondence: barbara.bonelli@polito.it
}

Received: 11 October 2019; Accepted: 14 October 2019; Published: 21 October 2019

Organics degradation is one of the challenges of Advanced Oxidation Processes (AOPs), which are mainly employed for the removal of water and air pollutants. Compared to stand-alone processes, AOPs are more effective if combined with other removal means, especially to degrade recalcitrant (stable) pollutants in subsequent steps.

Integrated systems able to solve the aforementioned issues in a single step could be less expensive and more efficient, but their development requires advancements from the point of view of both materials and the process. In this Issue, a system consisting of integrated resin adsorption/Dielectric Barrier Discharge (DBD) plasma regeneration was proposed to treat some textile dyes, showing that the DBD plasma could maintain the efficient adsorption performance of the resin while degrading the adsorbed dye [1].

Some AOPs imply the presence of catalyst, especially in photocatalytic processes: the goal of photocatalysis is to find efficient and stable materials (under the reaction conditions), which are able both to stabilize excitons (i.e., the generated electron/hole pairs) and to exploit solar light. However, the last two goals remain very ambitious and require breakthrough advances from the point of materials science (synthesis methods) and physical chemistry. Moreover, a multi-technique approach could help in studying the surface and textural properties of the photocatalyst in order to be able to unravel the phenomena regulating excitons formation and stabilization.

Different solutions are reported in the literature, including the production of nanocomposites [2,3] and of mixed phases of $\mathrm{TiO}_{2}$ [4]. The former have to be properly designed, like in Z-Scheme $\mathrm{g}-\mathrm{C}_{3} \mathrm{~N}_{4} / \mathrm{Fe}-\mathrm{TiO}_{2}$ [2] for the photodegradation of phenol, and in heterojunction nanostructured composites for photocatalytic fuel cells [3]: both systems were able to absorb in the Vis region. As a whole, the formation of heterojunctions in the nanocomposites simultaneously favors the photogenerated electron/hole separation and maintains the reduction and oxidation properties.

Occurrence of mixed phases is another means to promote and stabilize excitons, like in Degussa P25, where the mixed rutile/anatase phase is considered responsible for its good photocatalytic performance. Recently, mixed $\mathrm{TiO}_{2}$ phases containing brookite have been proved to display improved photocatalytic efficiency, like in N-doped anatase/brookite nanoparticles [4], obtained with high surface area by a low temperature sol-gel technique. Again, the development of new nanomaterials has been shown to have an impact on the progress of photocatalytic efficiency. Such advancements may be obtained by a plethora of synthesis methods, leading to different nanomaterials, like mixed Ni/Fe-based Metal Organic Frameworks (MOFs) [5] and Sr aluminates co-doped with Eu and Dy [6]. The former are porous networks, with high specific surface areas, where a thorough physico-chemical characterization by multiple techniques showed [5] that the occurrence of mixed-metal cluster $\mathrm{Fe}_{2} \mathrm{NiO}$ was able to enhance the photocatalytic performance further, via an electron transfer effect. The latter materials 
were instead prepared by different methods, namely with a hydrothermal reaction at low $\mathrm{T}$ and using a sol-gel method [6], pointing out the importance of developing new synthetic routes to obtain engineered (nano)materials for photocatalytic applications.

\section{References}

1. Hao, C.; Xiao, Z.; Xu, D.; Zhang, C.; Qiu, J.; Liu, K. Saturated Resin Ectopic Regeneration by Non-Thermal Dielectric Barrier Discharge Plasma. Catalysts 2017, 7, 362. [CrossRef]

2. Zhu, Z.; Murugananthan, M.; Gu, J.; Zhang, Y. Fabrication of a Z-Scheme g- $\mathrm{C}_{3} \mathrm{~N}_{4} / \mathrm{Fe}-\mathrm{TiO}_{2}$ Photocatalytic Composite with Enhanced Photocatalytic Activity under Visible Light Irradiation. Catalysts 2018, 8, 112. [CrossRef]

3. Pan, H.; Liao, W.; Sun, N.; Murugananthan, M.; Zhang, Y. Highly Efficient and Visible Light Responsive Heterojunction Composites as Dual Photoelectrodes for Photocatalytic Fuel Cell. Catalysts 2018, 8, 30. [CrossRef]

4. Jiang, L.; Li, Y.; Yang, H.; Yang, Y.; Liu, J.; Yan, Z.; Long, X.; He, J.; Wang, J. Low-Temperature Sol-Gel Synthesis of Nitrogen-Doped Anatase/Brookite Biphasic Nanoparticles with High Surface Area and Visible-Light Performance. Catalysts 2017, 7, 376. [CrossRef]

5. Nguyen, V.H.; Nguyen, T.D.; Bach, L.G.; Hoang, T.; Bui, Q.T.P.; Tran, L.D.; Nguyen, C.V.; Vo, D.N.; Do, S.T. Effective Photocatalytic Activity of Mixed Ni/Fe-Base Metal-Organic Framework under a Compact Fluorescent Daylight Lamp. Catalysts 2018, 8, 487. [CrossRef]

6. Park, B. Photocatalytic Behavior of Strontium Aluminates Co-Doped with Europium and Dysprosium Synthesized by Hydrothermal Reaction in Degradation of Methylene Blue. Catalysts 2018, 8, 227. [CrossRef]

(C) 2019 by the authors. Licensee MDPI, Basel, Switzerland. This article is an open access article distributed under the terms and conditions of the Creative Commons Attribution (CC BY) license (http://creativecommons.org/licenses/by/4.0/). 